\title{
problemas que plantea un horno Lepol sencillo para convertirlo en doble
}

La parrilla Lepol sencilla, consiste en una cadena formada por elementos metálicos ranurados para permitir el paso de gases. Envolviéndola existe una carcasa de chapa, forrada de material refractario, y en su parte inferior una tolva para recogida de gránulos y polvo perdidos en el proceso.

La cadena se acciona mediante un motor de velocidad variable, en nuestro caso concreto de corriente continua, que ataca un reductor acoplado al eje de accionamiento. Este lleva unas ruedas dentadas que engranan en la cadena.

Completan la instalación una artesa y la tolva de gránulos.

Un tabique colgado del techo divide la parte superior de la parrilla en dos cámaras: la primera, siguiendo el curso del material, llamada cámara preparatoria o de secado, y la segunda, denominada cámara caliente o de calcinación.

El polvo crudo llega a la artesa alimentado por un vis dosificador. Por la acción conjunta de un regado de agua y la rotación del plato se forman los gránulos, que caen a la tolva de alimentación. De ésta pasan a la parrilla, distribuyéndose uniformemente a todo lo ancho de la misma. La altura de la capa de gránulos se gradúa mediante una compuerta de altura regulable.

El material atraviesa la cámara de secado, a continuación pasan por la cámara de calcinación y luego al horno, donde acaba su proceso.

Los gases que proceden del horno, a una temperatura de $900-1.000^{\circ} \mathrm{C}$, se dividen en dos corrientes: Una que, mezclada con aire frío, hasta conseguir una temperatura de $200-300^{\circ} \mathrm{C}$, atraviesa la capa de gránulos en la cámara de preparación, efectuando el secado de los mismos; y otra, que atraviesa directamente la capa de la cámara caliente produciendo una decarbonatación parcial. En la parte inferior se mezclan ambas corrientes y abandonan la parrilla aspiradas por un ventilador que lo expulsa al exterior a una temperatura que oscila de 120 a $200^{\circ} \mathrm{C}$.

En el caso de doble paso de gases, el tabique de separación se prolonga por debajo de la cadena, dividiendo la parrilla en dos cámaras, tanto en la parte superior como la inferior de la misma.

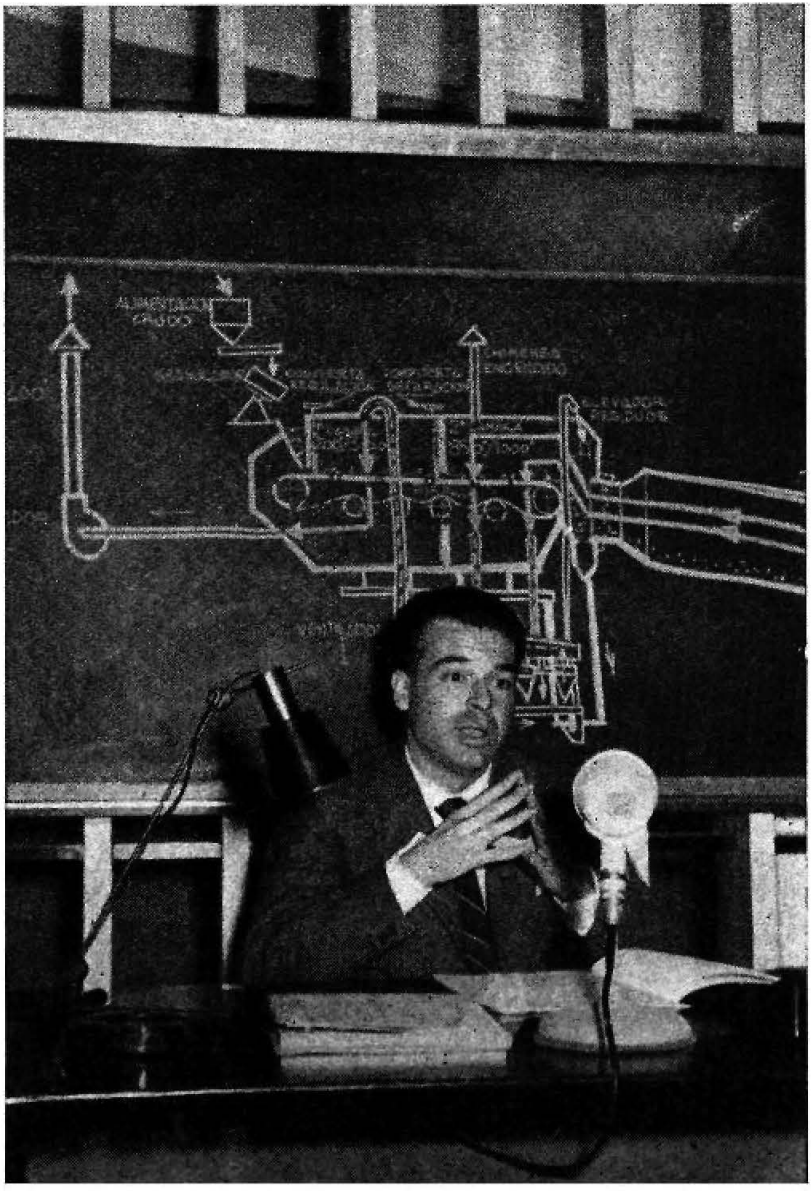


En este caso, todos los gases que provlenen del horno atraviesan la capa de la cámara callente. Aspirados por un ventilador intermedio, tras haber perdido gran parte del polvo arrastrado en unos ciclones colocados del lado de aspiración del ventilador, son impulsados a la cámara preparatorla, donde, aspirados por un segundo ventilador, atraviesan la capa de dicha cámara y son expulsados al exterior oscliando su temperatura entre $90-130^{\circ} \mathrm{C}$.

El objeto de los ciclones es evitar que el polvo arrastrado, llenando los huecos entre gránulos, ciegue el paso de gases.

Se ve inmediatamente que este sistema entraña una disminuclón en la cantidad y temperatura de los gases expulsados al evitar el paso directo al ventilador de gases procedentes de la cámara caliente, así como la adición de aire frío. Con ello tenemos la subsiguiente disminución del consumo caloríflco al disminuir las pérdidas en los gases de escape. Esta disminución se puede cifrar, aproximadamente, en $100 \mathrm{kcal} / \mathrm{kg}$ de clínker.

Al ser menor la cantidad de combustible, disminuye la cantidad de aire comburente, aumentando la temperatura del aire secundarlo, lo que aumenta la temperatura de la llama, mejorando el intercambio por radiación. Además, disminuye el volumen de gases, con lo cual bajan las pérdidas por radiación y convección.

Estas son las ventajas en cuanto se reflere a consumo calorífico. Por lo que respecta al arrastre de polvo, este dismfnuye considerablemente al hacer pasar todos los gases por eI lecho de gránulos húmedos, que, comportándose a modo de fltro, retiene gran parte del mismo. La cantidad de polvo arrastrado es, aproximadamente, un $1 \%$ de la producción.

Una ủltima ventaja del nuevo sistema es que al disminuir la cantidad de gases, disminuye la energia consumida por los ventiladores.

Abordemos ahora el caso concreto de Añorga. Nuestro problema principal era el arrastre de polvo, ya que nuestra situación, próxima a San Sebastian, nos ocasionaba numerosas molestias.

El horno del que tratamos data del año 1930, y es uno de los primeros construídos según el sistema Lepol. Consiste en un tubo de $3 \mathrm{~m} \varnothing$ por $30 \mathrm{~m}$ de longitud, y una parrilla de $3 \mathrm{~m} \times 10 \mathrm{~m}$. Lleva, además, un enfrtador Fuller tipo 629. Aparte del gran arrastre de polvo, los defectos princlpales de Ia parrilla eran su gran consumo de energia eléctrica y la enorme cantidad de aire falso que se introducía por las juntas de la misma.

Por todo ello, la soluclón, técnicamente ldeal, era construir una parrilla nueva. A esto se oponía el mayor costo de la instalación, y especialmente (tengamos en cuenta que estábamos en una época en ia que cuanto se producia se vendía) una mayor parada del horno. Estudiados los diversos puntos, se decidio hacer lo justamente imprescindible, aprovechando al máximo lo existente.

La modificación exigía prolongar la parrilla dos metros y, por lo tanto, desplazar la artesa y su depósito de altmentación.

Comenzamos por colocar una banda para poder alimentar la parrilla desde la artesa del otro horno. Hecho esto, se procedió al derribo del piso de la artesa. Construímos el nuevo y se colocó en su posición defnițiva ésta y su depósito. A continuación paramos el horno y se hizo todo el resto de la transformación, durando la parada doce días.

La puesta en mareha tuvo lugar sin diflcultades. Al principlo los hormeros se mostraban un tanto recelosos al tener que manejar más mandos. Su temor principal era el ventilador intermedio, ya que habia que vigilar su temperatura para evitar un agarrotamiento. Pasados los primeros días, vieron que sus temores eran infundados y que el manejo del horno era tan sencillo como podia serlo anteriormente.

Todo fué perfecto hasta que legamos a las $300 \mathrm{t} /$ dia. Las temperaturas de trabajo se mantenian, asi como las depresiones. Pero al llegar a esta producción, éstas subian de una manera anormal. El motlvo era que el gránulo, insuflelentemente preparado, estallaba al pasar de una cámara a otra, cegando el paso de gases. El horno marchaba ahogado, y la atmósfera reductora, unida a la gran cantidad de polvo que entraba en el mismo, nos ocaslonaba continuos anillos.

Para solucionario, aumentamos la temperatura en la cámara de preparación elevando el tabique de separación. Efectivamente, los gránulos ya no estallaban al pasar de una cámara a otra; pero... estallaban a la entrada de la parrilla; y todo seguia 1 gual.

Buscamos, entonces, la solución en aumentar la permeabilidad de los gránulos. Para ello se disminuyó su altura en la tolva de carga y aumentamos la Inclinación de la artesa.

Se logró una gran mejoría. Pero aún seguían estallando, si bien no con la intensidad de antes. 
Como última solución se colocó un segundo tabique en la cámara de secado, con objeto de conseguir, en la primera parte de la misma, una temperatura infertor a los $200^{\circ} \mathbf{C}$ que habiamos observado ser la critica.

Con esta modificación se acabó de normalizar el horno. Cesaron los estallidos y se pudo aumentar la producclón hasta $350 \mathrm{t}$, que es la que lleva hoy día.

Ya que hemos hablado de anillos, quisiera comen tarles algo sobre ello.

En un viaje que hice por Alemania, con miras al doble paso, observé que en la mayoria de las fábricas visitadas los tenian con bastante frecuencia. Esto nos hacia temer que el doble paso fuera muy propænso a su formación.

Los primeros inconvenientes con que se tropezó parecian confirmar este temor. Afortunadamente, se ha comprobado lo infindado de nuestras suposi ciones. No es que hayan desaparecido, aún se ciguen formando; pero no más que antes de la transforma ción.

Se forman a unos $12 \mathrm{~m}$ de la boca de salida. En el punto donde tlene lugar el depósito de cenizas.

Ha disminuído mucho la frecuencia de su iniciación variando en cada molienda la fnura del carbón, ya que de este modo desplazamos el depósito de cenizas. Cuando, asi y todo, tenemos principio de anillo lo combatimos, y generalmente con éxito, variando la posición del tubo de insuflación. Cuando esta medida no basta, se recurre a marchar unas horas con carbón más bajo en centzas y adíctonado de caliza,

Podemơs decir que prácticamente han desaparecido los anillos que obligan a parar el horno.

Otro tipo de acumulación que tenemos, es en el paso de la parrilla a horno. Cuando empleábamos solamente carbón no lo conociamos. Empezó con el fuel, y es' debido al azufre del mismo, que reaccio-

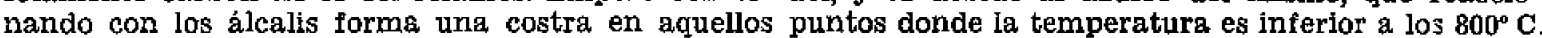
En el horno modificado ha disminuído la formación, pero es simplemente debido a que en él empleamos menos fuel.

De todas formas, no son muy molestos, pues basta limpiar semanalmente el conducto, cosa muy fácil de hacer en marcha con unas barras, para que nos olvidemos de su existencia.

Por lo que se reflere a la marcha actual del horno, se alimenta con crudo de un $35 \%$ de P. F. y un $12 \%$ de Ho de granulado.

El combustible empleado es una mezcla de carbón bajo en volátiles y fuel-oil. El consumo đe carbón es de un $11 \%$ de la producción de clínker, y el del fuel-oil de un 2,16\%, lo que da un consumo especiflco de $890 \mathrm{kcal}$. De éstas, 685 provienen del carbón y las 205 restantes del fuel-oll.

La temperatura de clinkerización es $-1.450^{\circ} \mathrm{C}$. En la parrilla tenemos $980^{\circ} \mathrm{C}$ en la cámara caliente, $280^{\circ} \mathrm{C}$ en Ia de preparación y $260^{\circ} \mathrm{C}$ en el ventilador intermedio, siendo de $108^{\circ} \mathrm{C}$ la de los gases de salida.

Las depresiones son:

En la cámara de preparación:

sobre la capa $=-31 \mathrm{~mm}$
debajo de la capa $=-73 \mathrm{~mm}$

En la cámara callente:

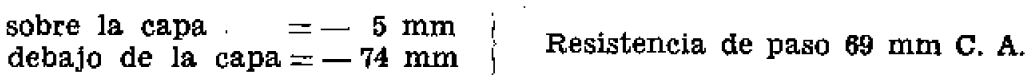

Observarán que existe una pequeña diferencla de depresión entre ambas cámaras en su parte inferior. Esta diferencia tiene un doble objeto: evitar que pase polvo directamente de la cámara caliente al ventilador de salida y mejorar el rendimiento térmiço al evitar ser expulsados al exterior gases a $250^{\circ} \mathrm{C}$. No conviene exagerar, porque sobrecargaríamos inútilmente el ventilador intermedio.

El material, al abandonar la parrilla, tiene un $27 \%$ de P. F., lo que supone una decarbonatación del $23 \%$, slendo su temperatura medla $680^{\circ} \mathrm{C}$.

El material recirculado, es decir, las pérdidas de la parrilla que se introducen directamente en el horno, asciende a $1.200 \mathrm{~kg} /$ hora. $O$ sea: un $8 \%$ de la producclón, siendo su temperatura media $315^{\circ} \mathrm{C}$.

El contenicio en $\mathrm{O}_{2}$ de los gases de combustión es del $2,2 \%$, equivalente a un $11 \%$ de exceso de aire, 10 que supone $1,1 \mathrm{~m}^{2} \mathrm{~N}$ de aire/kg de clinker. De éstos, el $15 \%$ como aire primario y el resto como aire secundario a $800^{\circ} \mathrm{C}$.

En la chimenea tenemos $2,6 \mathrm{~m}^{2} \mathrm{~N} / \mathrm{kg}$ de clinker, de los que 0,95 son alre falso introducido entre el hormo y la parrilla y debajo de la misma. Es una cantidad excesiva. El contenido en $\mathrm{O}_{2}$ pasa de 2,2 a $6 \%$ en el ventilador intermedio, y a $9 \%$ en la chimenea. 
Una mejora parcial, que realizaremos en la primera oportunidad, es camblar el anillo de cierre en la embocadura del horno con la parrilla. La total exigiría la sustitución de la parrilla por una de tipo moderno, que por caracteristicas constructivas, en cuyo detalle no vamos a entrar, presenta una hermeticidad muchisimo mayor.

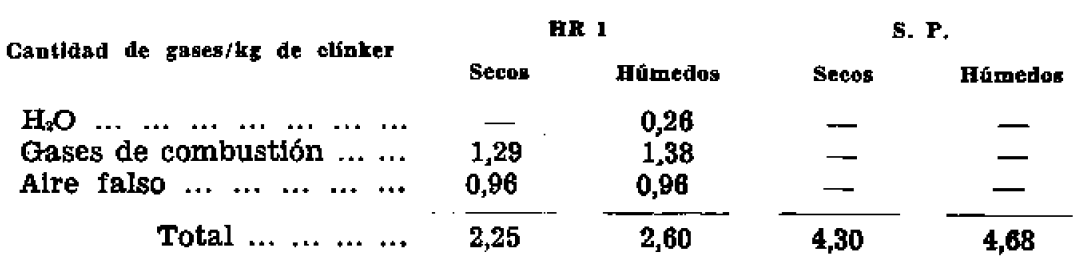

Como balance térmico simplificado podemos presentar el sigulente:

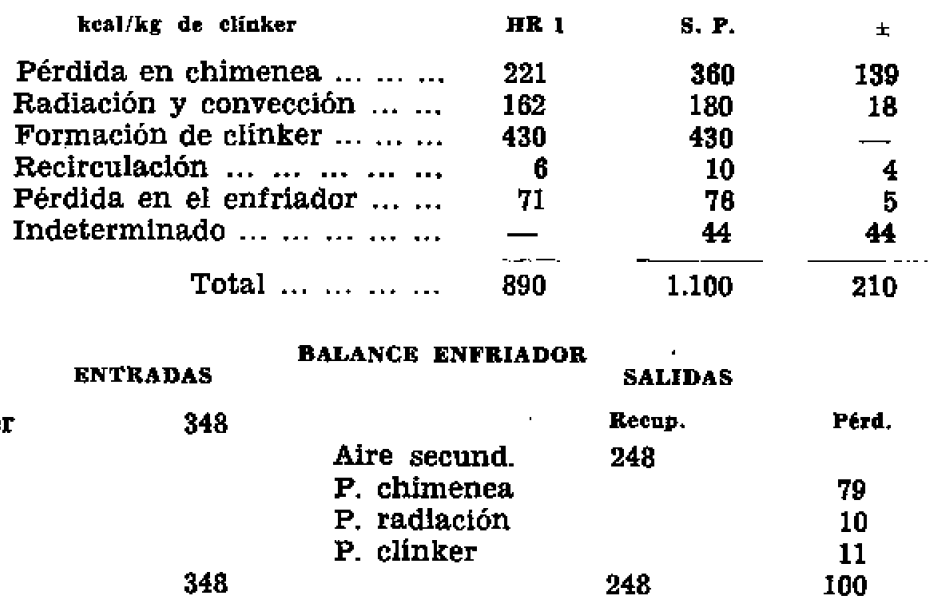

Clinker $=1.340^{\circ} \mathrm{C}(0,252)$, . Aire secundarlo $\left(0,94 \mathrm{~m}^{3} \mathrm{~N}\right) ; 800^{\circ} \mathrm{C}(0,328)$. - Aire en chimenea $\left(1,1 \mathrm{~m}^{8} \mathrm{~N}\right)$; $230^{\circ} \mathrm{C}(0,313)$.

El consumo especiflco de energía eléctrica es:

$\begin{array}{lccc}\text { kWh/toneladas de olinktr } & \text { HR } 1 & \text { S. F. } & \pm \\ \text { Total } & 14 & 15 & 1\end{array}$

Como mejoras posibles, veo las siguientes:

1. La ya indicada de disminución del aire falso. Sin pecar de optimistas, podemos suponer un contenido de $\mathrm{O}_{2}$ en los gases de escape del $7 \%$, que vendria a ser una ganancla de $\sim 10 \mathrm{kcal}$.

2. Emplear como aire primario el de escape de la chimenea del enfriador. Su temperatura seria $\sim 120^{\circ} \mathrm{C}$, con lo que el aire primario sería el $11 \%$. Ia ganancia sería $\sim 14 \mathrm{kcal} ; 4$ en el calor aportado por el aire primarlo, y 10 por el aumento de la cantidad de aire secundario.

3. Emplear los gases de escape del enfriador para secado del carbón que supondría $\sim 14$ kcal, y con lo que el consumo específlco quedaría en 837.

4. Aumentar la temperatura de trabajo del enfriador. Con lo que, si bien, aumentarian las pérdidas con el clínker y radiación, disminulrian en la chimenea. Podemos calcular unas 15 kcal.

Para terminar: El arrastre de polvo ha disminuido notablemente. Actualmente tenemos con electrofltro $0,206 \mathrm{~g} / \mathrm{m}^{3}$ de gas seco, lo que equivale a $0,178 \mathrm{~g} / \mathrm{m}^{2}$ de gas en la chimenea.

Sin electrofiltro: $3,70 \mathrm{~g} / \mathrm{m}^{3}$ de gas seco, algo menos del $1 \%$ de la producción.

Antes de la transformación los contenidos eran:

$1,425 \mathrm{~g} / \mathrm{m}^{\prime}$ con electrofiltro.

$10,65 \mathrm{~g} / \mathrm{m}^{\mathrm{x}} \sin$ electroflitro.

Datos, como ven, que no necesitan comentario.

Abierta la discusión, intervinieron los señores Torréns, De Bantiago, Calleja y Arteaga, comentando tres problemas básicos: recuperactón de polvo en los ciclones, depósito de sulfatos en la parrilla y marcha de los álcalis en los materiales en cocción. 